\title{
Block Factorization of Hankel Matrices and Euclidean Algorithm
}

\author{
S. Belhaj ${ }^{1,2 *}$ \\ ${ }^{1}$ Laboratoire de Mathématiques, CNRS UMR 6623, Université de Franche-Comté \\ 25030 Besançon cedex, France \\ ${ }^{2}$ Laboratoire LAMSIN, Ecole Nationale d'Ingénieurs de Tunis \\ BP 37, 1002 Tunis Belvédère, Tunisie
}

\begin{abstract}
It is shown that a real Hankel matrix admits an approximate block diagonalization in which the successive transformation matrices are upper triangular Toeplitz matrices. The structure of this factorization was first fully discussed in [1]. This approach is extended to obtain the quotients and the remainders appearing in the Euclidean algorithm applied to two polynomials $u(x)$ and $v(x)$ of degree $n$ and $m$, respectively, whith $m<n$.
\end{abstract}

Key words: block factorization, Hankel matrices, Toeplitz matrices, Euclidean algorithm AMS subject classification: 15A23, 15B05, 65F05, $11 \mathrm{C} 08$

\section{Introduction}

Let

$$
u(x)=\sum_{k=0}^{n} u_{k} x^{k} \text { and } v(x)=\sum_{k=0}^{m} v_{k} x^{k},
$$

be two polynomials in $\mathbb{R}[x]$ with $\operatorname{deg}(u(x))=n$, $\operatorname{deg}(v(x))=m$ and $m<n$. The classical Euclidean algorithm applied to $u(x)$ and $v(x)$ gives a sequence of polynomials quotients $q_{k}(x)$ and polynomials remainders $r_{k}(x)$, such that

$$
r_{-1}(x)=u(x), r_{0}(x)=v(x), r_{k-2}(x)=r_{k-1}(x) q_{k}(x)-r_{k}(x), \quad k=1, \ldots, K,
$$

where $-r_{k}(x)$ is the polynomial remainder of the division of $r_{k-2}(x)$ by $r_{k-1}(x)$ and $r_{K}(x)$ is the greatest common divisor (GCD) of $u(x)$ and $v(x)$.

The computation of the polynomials quotients and the polynomials remainders has been first studied in [6]. Bini and Gemignani (see $[4,5]$ ) have computed the coefficients of the polynomials

\footnotetext{
*Corresponding author. E-mail: skander.belhaj@univ-fcomte.fr
} 
in (1.1) via a new approach based on a block LU factorization (see $[8,9])$ of the Hankel matrix $H(u, v)$, associated to $u(x)$ and $v(x)$. More specifically,

$$
A^{t} H(u, v) A=D
$$

where $A$ is an upper triangular matrix with entries equal to one and $D$ is a block diagonal matrix with each block being a lower Hankel triangular matrix. Lately, Ben Atti and Diaz-Toca [7] are calculated the coefficients of the polynomials in (1.1) via a block LU factorization of the Hankel matrices different from the classical one [3]. Unfortunately, when the input polynomials are perturbed, the beautiful relation between the Euclidean algorithm and the block LU factorization of the Hankel matrices may be lost. That is why a new notion of the approximate block diagonalization has been introduced in [1] and its connection with the approximate Euclidean algorithm has been proposed in the same paper.

It is shown that a $n \times n$ real Hankel matrix admits an approximate block diagonalization in which the successive transformation matrices are upper triangular Toeplitz matrices.

The main contribution of this paper is to reveal the natural relation between the approximate Euclidean algorithm and the approximate block diagonalization [1] of the Hankel matrix associated to $u(x)$ and $v(x)$ and propose a revised algorithm which provide the approximate polynomials quotients and the approximate polynomials remainders during the processus execution.

The paper is organized as follows: Section 2 gives some theoretical results associated to Hankel matrices. A revised algorithm for an approximate block diagonalization of $n \times n$ real Hankel applied to two polynomials $u(x)$ and $v(x)$ of degree $n$ and $m$, respectively, with $m<n$ and its connection to the approximate Euclidean algorithm are introduced in Section 3. In Section 4 we illustrate our approach with an example given by an implementation of the procedures using Matlab. Finally, a summary and future research are given in Section 5 to complete the paper.

\section{Hankel matrices}

Let $u(x)=\sum_{k=0}^{n} u_{k} x^{k}$ and $v(x)=\sum_{k=0}^{m} v_{k} x^{k}$ be two polynomials in $\mathbb{R}[x]$ of degree $n$ and $m$, respectively, where $m<n$. The power series expansion $R(x)$ of the function $v(x) / u(x)$ at the infinity $R(x)=\sum_{k=0}^{\infty} h_{k} x^{-k}$ defines the $n \times n$ real Hankel matrix, $H=H(u, v)$, associated to $u(x)$ and $v(x)$,

$$
H=\left(\begin{array}{cccc}
h_{1} & h_{2} & \cdots & h_{n} \\
h_{2} & h_{3} & \cdots & h_{n+1} \\
\vdots & \vdots & \ddots & \vdots \\
h_{n} & h_{n+1} & \cdots & h_{2 n-1}
\end{array}\right)
$$

In addition, every nonsingular real Hankel matrix can be viewed as a Hankel matrix associated to two polynomials.

Proposition 1. For any nonsingular $n \times n$ Hankel matrix $H$ there exists two coprime polynomials $u(x)$ and $v(x)$ of degree $n$ and $m$, respectively, where $m<n$, such that $H=H(u, v)$. The 
polynomial $u(x)$ and $v(x)$ are related to $H$ by the following equalities:

$$
H\left(u_{0}, \ldots, u_{n-1}\right)^{t}=-u_{n}\left(h_{n+1}, \ldots, h_{2 n}\right)^{t},\left(v_{n-1}, \ldots, v_{0}\right)^{t}=l T\left(h_{1}, \ldots, h_{n}\right)\left(u_{n}, \ldots, u_{1}\right)^{t},
$$

where $h_{2 n}$ is any number and $l T\left(h_{1}, \ldots, h_{n}\right)$ is the lower triangular Toeplitz matrix defined by the list $\left[h_{1}, \ldots, h_{n}\right]$.

Proof. (See [5]).

Remark 2. Suppose that $H$ has the following structure: $H=l H\left(h_{n}, \ldots, h_{2 n-1}\right):$ a lower triangular Hankel matrix defined by the list $\left[h_{n}, \ldots, h_{2 n-1}\right]$. Thus, Proposition1 concludes, for any polynomial $v(x)$,

$$
\left(v_{n-1}, \ldots, v_{0}\right)^{t}=\left(\begin{array}{ccc}
0 & & \\
\vdots & \ddots & \\
h_{n} & \cdots & 0
\end{array}\right)\left(u_{n}, \ldots, u_{1}\right)^{t}=\left(0, \ldots, 0, h_{n} u_{n}\right)^{t} .
$$

If $v(x)=1$ then $v_{0}=1$ and $u_{n}=1 / h_{n}$. Thus, $H$ represents a Hankel matrix associated to $u(x)$ and $v(x)=1: H=H(u, 1)$.

Moreover we will use the following notations.

- $u T(S) \in \mathbb{R}^{n \times n}$ denotes the upper Toeplitz triangular matrix associated to a list $S$ such that the first row is defined by $S$.

- $l H(S) \in \mathbb{R}^{n \times n}$ denotes the lower Hankel triangular matrix (with respect to the antidiagonal) associated to a list $S$ of length $(2 n-1)$ such that the last column is defined by $S$.

- Let $p \in \mathbb{N}$. Let $\Sigma_{p} \in \mathbb{R}^{p \times p}, \Sigma_{p}=\left[\varepsilon_{j k}\right]_{j, k=1}^{p}$, where all entries of $\Sigma_{p}$ are zero except that $\varepsilon_{j+k, j}=\varepsilon_{k}$ for $j, k=1,2, \ldots, p$.

- Given $P \in \mathbb{R}^{n \times m}, \widetilde{P}=J_{m} P^{t} J_{n}$ where $J_{p}=l H(1, \underbrace{0, \ldots, 0}_{p-1}), p \in \mathbb{N}$.

- Let $a \in \mathbb{R}$. Let $\mu>0, V(a, \mu)=(a-\mu ; a+\mu)$ is a neighborhood of $a$.

Lemma 3. Let $n \in \mathbb{N}^{*}$. Let $h=H\left(h_{1}, \ldots, h_{2 n-1}\right)$ be a square Hankel matrix of order $n$. Suppose that $h_{j}=\varepsilon_{j}$ with $\varepsilon_{j} \in V(0, \mu)$ for $j=1,2, \ldots, p-1$ and $h_{p} \notin V(0, \mu)$. Then $h$ has the form

$$
h=H\left(\varepsilon_{1}, \ldots, \varepsilon_{p-1}, h_{p}, \ldots, h_{2 n-1}\right)=\left(\begin{array}{ll}
h_{11} & h_{12} \\
h_{21} & h_{22}
\end{array}\right),
$$

where

$$
\begin{gathered}
h_{11}=l H\left(h_{p}, \ldots, h_{2 p-1}\right)+J_{p} \Sigma_{p}, \quad h_{22}=H\left(h_{2 p+1}, \ldots, h_{2 n-1}\right), \\
h_{12}=H\left(h_{p+1}, \ldots, h_{n+p-1} ; p ; n-p\right), \quad h_{21}=h_{12}^{t} .
\end{gathered}
$$


We can successively construct from $h$ the following two matrices:

- A square lower Hankel triangular matrix $H$ of order $(2 n-p)$,

$$
\mathcal{H}=l H\left(h_{p}, \ldots, h_{2 n-1}\right)=\left(\begin{array}{ccc}
0 & 0 & H_{13} \\
0 & h_{11} & h_{12} \\
H_{31} & h_{12}^{t} & h_{22}
\end{array}\right)
$$

where $H_{31}=H_{13}=l H\left(h_{p}, \ldots, h_{n-1}\right)$.

- A square upper triangular Toeplitz matrix $T$,

$$
T=J_{2 n-r} \mathcal{H}=u T\left(h_{p}, \ldots, h_{2 n-1}\right)=\left(\begin{array}{ccc}
t_{11} & t_{12} & t_{13} \\
0 & t_{22} & t_{23} \\
0 & 0 & t_{33}
\end{array}\right)
$$

where $t_{k j}=J H_{3-i+1, j}, t_{11}=t_{33}$ and $t_{12}=\widetilde{t}_{23}$.

Lemma 4. Let $T$ be an upper triangular matrix, nonsingular with non-zero diagonal. Then $T^{-1}=$ $u T\left(\mu_{1}, \ldots, \mu_{2 n-p}\right)$ and has the following block decomposition,

$$
T^{-1}=\left(\begin{array}{ccc}
\left(T^{-1}\right)_{11} & \left(T^{-1}\right)_{12} & \left(T^{-1}\right)_{13} \\
0 & \left(T^{-1}\right)_{22} & \left(T^{-1}\right)_{23} \\
0 & 0 & \left(T^{-1}\right)_{33}
\end{array}\right)=\left(\begin{array}{ccc}
t_{11}^{-1} & \widetilde{P} & Q \\
0 & t_{22}^{-1} & P \\
0 & 0 & t_{11}^{-1}
\end{array}\right)
$$

where

$$
\begin{gathered}
P=T\left(\mu_{2}, \ldots, \mu_{n} ; p ; n-p\right), \quad \widetilde{P}=J_{n-p} P^{t} J_{r}, \\
t_{22} P+t_{23} t_{11}^{-1}=0_{(p, n-p)}, \quad h_{11} P+M t_{11}^{-1}=0_{(p, n-p)}, \\
t_{11} \widetilde{P}+\widetilde{t}_{23} t_{22}^{-1}=0_{(n-p, p)}, \quad t_{11} Q+\widetilde{t}_{23} P+t_{13} t_{11}^{-1}=0_{(n-p, n-p)} .
\end{gathered}
$$

\section{Approximate block diagonalization for $H(u, v)$}

In this section, we introduce the correlation between the approximate Euclidean algorithm applied to two polynomials $u(x)$ and $v(x)$ and the approximate block diagonalization of a Hankel matrix. From Remark 2 it follows that Theorem 5 of [1] can be rewritten in terms of Hankel matrix associated with $u(x)$ and $v(x)$.

Theorem 5. Let $H(u, v)=H\left(\varepsilon_{1}, \ldots, \varepsilon_{n-m-1}, h_{n-m}, \ldots, h_{2 n-1}\right)$ an approximate Hankel matrix associated to two coprime polynomials in $\mathbb{R}[x], u(x)=\sum_{k=0}^{n} u_{k} x^{k}$ and $v(x)=\sum_{k=0}^{m} v_{k} x^{k}$, $\operatorname{deg}(u(x))=n, \operatorname{deg}(v(x))=m$ and $m<n$, where $\varepsilon_{j} \in V(0, \mu)$ for $j=1,2, \ldots, n-m-1$ with $h_{n-m} \notin V(0, \mu)$. Let

$$
T=u T\left(h_{n-m}, \ldots, h_{2 n-1}\right), \quad T^{-1}=u T\left(\mu_{1}, \ldots, \mu_{n-m}\right),
$$




$$
t=u T\left(h_{n-m}, \ldots, h_{2 n-m-1}\right), \quad t^{-1}=u T\left(\mu_{1}, \ldots, \mu_{n}\right)
$$

Then

$$
\left(t^{-1}\right)^{t} H(u, v) t^{-1}=\left(\begin{array}{cc}
\widetilde{H}(q, 1) & \epsilon^{\prime} \\
\left(\epsilon^{\prime}\right)^{t} & \widetilde{H}(v, r)
\end{array}\right)
$$

where

$$
\begin{gathered}
\widetilde{H}(q, 1)=H(q, 1)+\left(t_{22}^{-1}\right)^{t} J_{n-m} \Sigma_{n-m} t_{22}^{-1}, \\
\widetilde{H}(v, r)=H(v, r)+P^{t} J_{n-m} \Sigma_{n-m} P, \epsilon^{\prime}=\left(t_{22}^{-1}\right)^{t} J_{n-m} \Sigma_{n-m} P,
\end{gathered}
$$

$q(x)$ and $r(x)$ are the polynomial quotient and remainder of the division $u(x) / v(x)$.

Proof. $H(u, v)$ is defined by the first $(2 n-1)$ terms of $\frac{v(x)}{u(x)}=\sum_{k=0}^{\infty} h_{k} x^{-k}$ and so $\left(u T\left(h_{n-m}, \ldots, h_{2 n-1}\right)\right)^{-1}$ is given by the first $(n-m)$ terms of

$$
\frac{u(x)}{v(x)}=\mu_{1} x^{n-m}+\cdots+\mu_{n-m+1}+\sum_{k=1}^{\infty} \mu_{n-m+1+k} x^{-k}=q(x)-\frac{r(x)}{v(x)} .
$$

Then,

$$
\begin{aligned}
& h_{11}^{\prime}=l H\left(\mu_{1}, \ldots, \mu_{n-m}\right)+\left(t_{22}^{-1}\right)^{t} J_{n-m} \Sigma_{n-m} t_{22}^{-1}=H(q, 1)+\left(t_{22}^{-1}\right)^{t} J_{n-m} \Sigma_{n-m} t_{22}^{-1} . \\
& h_{22}^{\prime}=-H\left(\mu_{n-m+2}, \ldots, \mu_{n+m}\right)+P^{t} J_{n-m} \Sigma_{n-m} P=H(v, r)+P^{t} J_{n-m} \Sigma_{n-m} P .
\end{aligned}
$$

Let us devise the algorithm for the computation of the approximate polynomials quotients and the approximate polynomials remainders appearing in the approximate Euclidean algorithm applied to two polynomials $u(x)$ and $v(x)$ of degree $n$ and $m$, respectively, $m<n$.

Algorithm 6. (Approximate block diagonalization for $H(u, v)$ ) Given $u(x)=\sum_{k=0}^{n} u_{k} x^{k}$ and $v(x)=\sum_{k=0}^{m} v_{k} x^{k}$ two polynomials in $\mathbb{R}[x]$ of degree $n$ and $m$, respectively, where $m<n$, this algorithm computes the approximate polynomials quotients and the approximate polynomials remainders appearing in the Euclidean Algorithm with accuracy less than a small positive number $\epsilon, 0<\epsilon \ll 1$.

1. Construct $H=H\left(\varepsilon_{1}, \ldots, \varepsilon_{n-m-1}, h_{n-m}, \ldots, h_{2 n-1}\right)$ (The first step : $\varepsilon_{1}=\cdots=\varepsilon_{n-m-1}=$ $0)$.

2. Define an upper triangular Toeplitz matrix $t=u T\left(h_{n-m}, \ldots, h_{2 n-m-1}\right)$.

3. Compute $t^{-1}$ and $h^{\prime}=\left(t^{-1}\right)^{t} h t^{-1}$.

4. Set $h_{11}^{\prime}=h^{\prime}(1: n-m, 1: n-m)$ and $h_{22}^{\prime}=h^{\prime}(n-m+1: n, n-m+1: n)$.

5. Recover the coefficients of the quotient polynomial from step 3.

6. Recover the coefficients of the remainder polynomial from (3.3).

7. Recursively apply Algorithm 3.1 to $h=H\left(h_{22}^{\prime}(1: m, 1) h_{22}^{\prime}(m, 1: m)\right)$, obtaining all the coefficients appearing in the approximate Euclidean algorithm. 


\section{Numerical example}

The example in this section is taken from [7]. We introduce input polynomials via a perturbation (add $k \cdot 10^{-13}$ to all entries of exact input polynomials with $k$ is taken randomly in $(0,1)$ ) of another exact input polynomials, whose sequence of polynomials quotients and polynomials remainders is exactly known. The errors of the sequences of quotients and remainders between the approximate Euclidean algorithm and our method are, respectively:

$$
\operatorname{Error}_{Q}=\left\|Q_{\text {Our approx }}-Q_{\text {approx EA }}\right\|_{1}, \operatorname{Error}_{R}=\left\|R_{\text {Our approx }}-R_{\text {approx EA }}\right\|_{1} .
$$

Let $u(x)=6 x^{9}+24 x^{8}+44 x^{7}+162 x^{6}+60 x^{5}+273 x^{4}+32 x^{3}+193 x^{2}-70 x-10$, and $v(x)=2 x^{7}+6 x^{6}+6 x^{5}+40 x^{4}-28 x^{3}+65 x^{2}-19 x-2$.

In the following, we show the parallelism between the approximate block factorization for $H(u, v)$ and the approximate Euclidean Algorithm (for every steps).

Step 1: Approximate block factorization for $H(u, v)$

$H\left(q_{1}, 1\right)=H(0,3,3)$,

$H\left(v, r_{1}\right)=H(-5.6 e-15,1.2 e-14,-1,2.2 e-13,5,-1,-25,10.5,124,-81,-609,545,2968.2)$,

$q_{1}(x)=3 x^{2}+3 x+3.9999$,

$r_{1}(x)=-2.0000 x^{4}-5.9999 x^{3}+3.9999 x^{2}-12 x+1.9999$.

Step 1: Approximate Euclidean algorithm

$r_{-1}(x)=r_{0}(x) q_{1}(x)-r_{1}(x), q_{1}(x)=\mathbf{2 . 9 9 9 9} x^{2}+\mathbf{3} x+\mathbf{3 . 9 9 9 9}$,

$r_{1}(x)=-2.0000 x^{4}-5.9999 x^{3}+3.9999 x^{2}-11.9999 x+2.0000$.

Step 2: Approximate block factorization for $H(u, v)$

$H\left(q_{2}, 1\right)=H(-5.6 e-15,1.2 e-14, \mathbf{- 1}, \mathbf{- 2 . 2 e - 1 3}, \mathbf{- 5})$,

$H\left(r_{1}, r_{2}\right)=H(-2.3 e-11,0.5,-3.2 e-10,-1,-5.3 e-9,-1.5,10.5)$,

$q_{2}(x)=-0.9999 x^{3}-2.2019 \mathrm{e}-13 x^{2}-4.9999 x+0.9999$,

$r_{2}(x)=-1.0000 x^{2}-3.0000 x+3.9999$.

Step 2: Approximate Euclidean algorithm

$r_{0}(x)=r_{1}(x) q_{2}(x)-r_{2}(x), q_{2}(x)=-0.9999 x^{3}+3.7334 \mathrm{e}-11 x^{2}+-4.9999 x+0.9999$,

$r_{2}(x)=-0.9999 x^{2}-2.9999 x+3.9999$.

Step 3: Approximate block factorization for $H(u, v)$

$H\left(q_{3}, 1\right)=H(-9.5-11,2, \mathbf{1 . 2 e - 9})$,

$H\left(r_{2}, r_{3}\right)=H(-2.6 e-8,-14,42)$,

$q_{3}(x)=1.9999 x^{2}+1.2842 \mathrm{e}-9 x+3.9999, r_{3}(x)=13.9999$.

Step 3: Approximate Euclidean algorithm

$r_{1}(x)=r_{2}(x) q_{3}(x)-r_{3}(x), q_{3}(x)=1.9999 x^{2}-3.5625 \mathrm{e}-8 x+3.9999, r_{3}(x)=13.9999$.

We also present the errors between the approximate Euclidean algorithm and our method in the following table :

\begin{tabular}{|l|c|c|}
\hline Steps & Error $_{Q}$ & Error $_{R}$ \\
\hline 1 & $4.884981308350689 \times 10^{-15}$ & $8.546496843564455 \times 10^{-13}$ \\
\hline 2 & $2.360093676041454 \times 10^{-09}$ & $4.352333782264850 \times 10^{-08}$ \\
\hline 3 & $6.247234852658265 \times 10^{-07}$ & $2.142632679280609 \times 10^{-06}$ \\
\hline
\end{tabular}




\section{Conclusion}

In this paper, we addressed the task of computing the approximate polynomials quotients and the approximate polynomials remainders appearing in the approximate Euclidean algorithm applied to two polynomials $u(x)$ and $v(x)$ of degree $n$ and $m$, respectively, $m<n$ with real coefficients via a new approach based on the block diagonalization of a Hankel matrix $H(u, v)$. To allow better numerical stability, an approximate Schur-based method is reformulated in [2] in terms of an $n \times n$ real Bézout matrix $B(u, v)$ associated to the input polynomials for computing the coefficients of the polynomials generated by the approximate Euclidean algorithm.

\section{References}

[1] S. Belhaj. A fast method to block-diagonalize a Hankel matrix. Numer Algor, 47 (2008), 15-34.

[2] S. Belhaj. Block diagonalization of Hankel and Bézout matrices : connection with the Euclidean algorithm, submitted.

[3] N. Ben Atti, G.M. Diaz-Toca. Block diagonalization and LU-equivalence of Hankel matrices. Linear Algebra and its Applications, 412 (2006), 247-269.

[4] D. Bini, L. Gemignani. On the Euclidean scheme for polynomials having interlaced real zeros. Proc. 2nd ann. ACM symp. on parallel algorithms and architectures, Crete, (1990), 254-258.

[5] D. Bini, L. Gemignani. Fast parallel computation of the polynomial remainder sequence via Bézout and Hankel matrices. SIAM J. Comput., 24 (1995), 63-77.

[6] A. Borodin, J. Von Zur Gathen, J. Hopcroft. Fast parallel matrix and ged computation. Information and Control, 52 (1982), 241-256.

[7] G. Diaz-Toca, N. Ben Atti. Block LU factorization of Hankel and Bezout matrices and Euclidean algorithm. Int. J. Comput. Math., 86 (2009), 135-149.

[8] W. B. Gragg, A. Lindquist. On partial realization problem. Linear Algebra Appl., 50 (1983), 277-319.

[9] G. Heining, K. Rost. Algebraic methods for Toeplitz-like matrices and operators. Birkhäuser Verlag, Basel, 1984. 